\title{
Magnetic field dependence of muonium-antimuonium conversion
}

\author{
Wei-Shu Hou, Gwo-Guang Wong \\ Department of Physics, National Taiwan University, Taipei, Taiwan 10764, ROC
}

Received 22 May 1995; revised manuscript received 5 July 1995

Editor: H. Georgi

\begin{abstract}
We study the magnetic field dependence of muonium-antimuonium conversion for all relevant operators, such as ( $V$ $A)(V-A),(V-A)(V+A), S S$ and $P P$. The traditional $(V-A)(V-A)$ interaction suffers two stage quenching and becomes strongly suppressed by $10^{3}$ Gauss fields or higher. In contrast, all other operator types (except $(S-P)(S-P)$ ) have a weaker field dependence and in general are not strongly quenched by strong fields. Since ongoing experiments are done with sizable magnetic fields, our results are useful for the interpretation of experimental results as bounds on different effective interactions.
\end{abstract}

The spontaneous conversion of muonium (hydrogen-like atom $M=\mu^{+} e^{-}$) into antimuonium $(\bar{M})$ would violate the separate additive muon and electron numbers, but would remain consistent with multiplicative muon or electron number conservation [1]. It is traditional to consider an effective $(V-A)(V-A)$ interaction,

$$
\mathcal{H}_{M \bar{M}}=\frac{G_{M \bar{M}}}{\sqrt{2}} \bar{\mu} \gamma_{\lambda}\left(1-\gamma_{5}\right) e \bar{\mu} \gamma^{\lambda}\left(1-\gamma_{5}\right) e+\text { h.c. }
$$

The existing limit on $G_{M \bar{M}}$ is $0.16 G_{F}$ [2], where $G_{F}$ is the Fermi constant. An order of magnitude improvement has just been reported [3], namely

$$
G_{M \bar{M}}<1.8 \times 10^{-2} G_{F}
$$

with the ultimate aim [4] of reaching the level of $10^{-3} G_{F}$. The subject is therefore of continued experimental interest.
The $(V-A)(V-A)$ interaction of Eq. (1) is motivated by doubly charged scalars in left-right symmetric models [5], where the effective interaction is of $(V \pm A)(V \pm A)$ form after Fierz rearrangement. Effective $(V-A)(V+A)$ interactions [6] can arise via dilepton gauge bosons [7], while neutral scalar and pseudoscalar models [8] (see also [9,10]) could lead to effective $S S$ and $P P$ operators. Thus, possible operator forms are far richer than that of Eq. (1). Experimental limits on these different interactions have not yet been given.

Recently, Horikawa and Sasaki [11] pointed out that $(V-A)(V+A)$ interactions are considerably less sensitive to magnetic fields compared to the ( $V-$ $A)(V-A)$ case. Since actual experiments are conducted in the presence of sizable magnetic fields [24], the implication is that one has to be careful when interpreting the bound of Eq. (2) for interactions other than that of Eq. (1). In this Letter we study the magnetic field dependence of all relevant $M-\bar{M}$ conversion 
operators.

To explore the magnetic field dependence of muonium conversion probabilities, consider the Hamiltonian for $1 S$ muonium,

$\mathcal{H}=\mathcal{H}_{0}+a s_{e} \cdot \boldsymbol{s}_{\bar{\mu}}-\boldsymbol{\mu}_{e} \cdot \boldsymbol{B}-\boldsymbol{\mu}_{\bar{\mu}} \cdot \boldsymbol{B}+\mathcal{H}_{M \bar{M}}$,

where $\mathcal{H}_{0}$ gives the $1 S$ energy $E_{0}=-\alpha^{2} m / 2$, with reduced mass $1 / m=1 / m_{e}+1 / m_{\mu}, a \cong 1.846 \times 10^{-5}$ $\mathrm{eV}$ is the $1 S$ muonium hyperfine splitting, and $\boldsymbol{\mu}_{e}=$ $-g_{e} \mu_{B} s_{e}, \mu_{\bar{\mu}}=+g_{\mu} \mu_{B} \frac{m_{e}}{m_{\mu}} s_{\mu}$, where $g_{e} \cong g_{\mu} \cong$ 2 and $\mu_{B}=e /\left(2 m_{e}\right) \cong 5.788 \times 10^{-9} \mathrm{eV} /$ Gauss is the Bohr magneton. Introducing the dimensionless parameters

$X, Y \equiv \frac{\mu_{B} B}{a}\left(g_{e} \pm \frac{m_{e}}{m_{\mu}} g_{\mu}\right)$,

we see that $|Y|$ is just $1 \%$ smaller than $|X|$. Ignoring $\mathcal{H}_{M \bar{M}}$ for the moment, the four muonium Breit-Rabi energy levels [12] are

$E_{M}(1, \pm 1)=E_{0}+\frac{a}{2}\left(\frac{1}{2} \pm Y\right)$,

$E_{M}(1,0)=E_{0}+\frac{a}{2}\left(-\frac{1}{2}+\sqrt{1+X^{2}}\right)$,

$E_{M}(0,0)=E_{0}+\frac{a}{2}\left(-\frac{1}{2}-\sqrt{1+X^{2}}\right)$,

which correspond to the eigenstates

$|M ; 1, \pm 1\rangle=|M ; \uparrow \uparrow\rangle,|M ; \downarrow \downarrow\rangle$,

$|M ; 1, \quad 0\rangle=c|M ; \uparrow \downarrow\rangle+s|M ; \downarrow \uparrow\rangle$,

$|M ; 0, \quad 0\rangle=-s|M ; \uparrow \downarrow\rangle+c|M ; \downarrow \uparrow\rangle$,

where the magnetic field dependent "rotation" is

$s=\frac{1}{\sqrt{2}}\left[1-\frac{X}{\sqrt{1+X^{2}}}\right]^{\frac{1}{2}}$,

$c=\frac{1}{\sqrt{2}}\left[1+\frac{X}{\sqrt{1+X^{2}}}\right]^{\frac{1}{2}}$.

We have labeled the Breit-Rabi energy levels with the weak field basis $\left|M ; F, m_{F}\right\rangle$, i.e. the corresponding zero $B$ field $(X, Y \rightarrow 0$ and $s, c \rightarrow 1 / \sqrt{2}$ ) hyperfine states. The "uncoupled" basis of $\left|M ; m_{s_{e}}, m_{s_{\bar{\mu}}}\right\rangle$ corresponds to the strong field limit of $X, Y \gg 1$ and $s \rightarrow 0$.
In this limit, $|M ; 1,0\rangle \rightarrow|M ; \uparrow \downarrow\rangle$ and $|M ; 0,0\rangle \rightarrow$ $|M ; \downarrow \uparrow\rangle$, and the electron and muon hypcrfine spinspin coupling is overwhelmed by the Zeeman effect.

For antimuonium, again ignoring the effect of $\mathcal{H}_{M \bar{M}}$, retaining the spin labels and with uncoupled basis $\left|\bar{M} ; m_{s_{\bar{e}}}, m_{s_{\mu}}\right\rangle$, one simply flips $X \rightarrow-X, Y \rightarrow-Y$ and hence interchange $s \leftrightarrow c$ in Eqs. (6) and (7). The notable changes are

$E_{\bar{M}}(1, \mp 1)=E_{M}(1, \pm 1)$,

since for given spin, the antiparticle magnetic moments have flipped sign, and

$$
\begin{aligned}
& |\bar{M} ; 1,0\rangle=s|\bar{M} ; \uparrow \downarrow\rangle+c|\bar{M} ; \downarrow \uparrow\rangle, \\
& |\bar{M} ; 0,0\rangle=-c|\bar{M} ; \uparrow \downarrow\rangle+s|\bar{M} ; \downarrow \uparrow\rangle .
\end{aligned}
$$

We have the following energy differences between $M$ and $\bar{M}$ eigenstates:

$$
\begin{aligned}
& E_{M}(1, \pm 1)-E_{\bar{M}}(1, \pm 1)= \pm a Y, \\
& E_{M}(1,0)-E_{\bar{M}}(1,0) \\
& \quad=E_{M}(0,0)-E_{\bar{M}}(0,0)=0, \\
& E_{M}\left(\begin{array}{ll}
1, & 0)-E_{\bar{M}}(0,0) \\
\quad=-\left(E_{M}(0,0)-E_{\bar{M}}(1,0)\right)=a \sqrt{1+X^{2}} .
\end{array}\right.
\end{aligned}
$$

The effect of $\mathcal{H}_{M \bar{M}}$ is treated as a perturbation. Define generically

$\left\langle\bar{M}\left|\mathcal{H}_{M \bar{M}}\right| M\right\rangle=\delta / 2$

(for simplicity, we take $\delta$ to be real) between any two Breit-Rabi $M$ and $\bar{M}$ energy eigenstates, it was shown by Feinberg and Weinberg [1] that the time integrated probability for an initial muonium state to decay as antimuonium is

$P(\bar{M})=\frac{\delta^{2}}{2\left(\delta^{2}+\Delta^{2}+\lambda^{2}\right)}$,

where $\Delta=E_{M}-E_{\bar{M}}$ is the energy difference, and $\lambda \cong 2.996 \times 10^{-10} \mathrm{eV}$ is the muon decay rate. The physics is clear: muonium oscillation has to compete with muon decay and the damping from oscillations between two states that are very disparate in energy. The total transition probability is

$P_{T}(\bar{M})=\sum_{F, m_{r}}\left|c_{F, m_{F}}\right|^{2} P^{\left(F, m_{F}\right)}(\bar{M})$ 
where $\left|c_{F, m_{F}}\right|^{2}$ are the populations in muonium states of Eq. (6), and $P^{\left(F, m_{F}\right)}(\bar{M})$ are the probabilities for an initial $\left(F, m_{F}\right)$ muonium state to decay as antimuonium.

In principle $P^{(1,0)}(\bar{M})$ and $P^{(0,0)}(\bar{M})$ also contain the probabilities of initial $(1,0)$ or $(0,0)$ muonium states to decay as $(0,0)$ or $(1,0)$ antimuonium, respectively, since $(1,0)$ and $(0,0)$ are mixtures of unpolarized states. To show that these are vanishingly small, it is useful to notice the hierarchy

$\delta \ll \lambda \ll a$,

the first of which follows from Eq. (2) for any $\mathcal{H}_{M \bar{M}}$ model. Combining Eqs. (10) and (12) we see that $(1,0) \rightarrow(0,0)$ and $(0,0) \rightarrow(1,0)$ transitions are extremely suppressed by hyperfine splitting even for $B=0$, and need not be considered. Similarly, although $(1, \pm 1) \rightarrow(1, \pm 1)$ transitions contribute in $B=0$ limit, they become rapidly suppressed even for rather weak magnetic fields [1], because of the mismatch between Zeeman energy levels for $M$ vs. $\bar{M}$ polarized states with same $m_{F}$.

In this work we will discuss only the relative magnetic field dependence of $M-\bar{M}$ conversion probabilities. Differences in coupling strength for various effective operators at zero $B$ field can be found in Refs. $[5,6,8]$. Hence, we normalize effective interactions to the conversion probability due to Eq. (1) at zero magnetic field, and in particular for equally populated (1/4 each) Breit-Rabi states (the latter condition would be removed at the end). Thus, the zero field total transition probability is

$P_{T}(\bar{M} ; B=0) \simeq \frac{\bar{\delta}^{2}}{2 \lambda^{2}}=2.56 \times 10^{-5}\left(\frac{G_{M \bar{M}}}{G_{F}}\right)^{2}$,

which defines the parameter $\bar{\delta}$.

For the $(V-A)(V-A)$ case one basically multiplies the r.h.s. (right hand side) of Eq. (11) by $\delta_{m_{s_{p}} m_{s_{e}}} \delta_{m_{s_{\mu}} m_{s_{\tilde{\mu}}}}$, hence

$$
\begin{aligned}
& \left\langle\bar{M} ; 1, \pm 1\left|\mathcal{H}_{M \bar{M}}\right| M ; 1, \pm 1\right\rangle=\frac{\bar{\delta}}{2} \\
& \left\langle\bar{M} ; 1,0\left|\mathcal{H}_{M \bar{M}}\right| M ; 1,0\right\rangle=\left\langle\bar{M} ; 0,0\left|\mathcal{H}_{M \bar{M}}\right| M ; 0,0\right\rangle \\
& \quad=\frac{\bar{\delta}}{2 \sqrt{1+X^{2}}}
\end{aligned}
$$

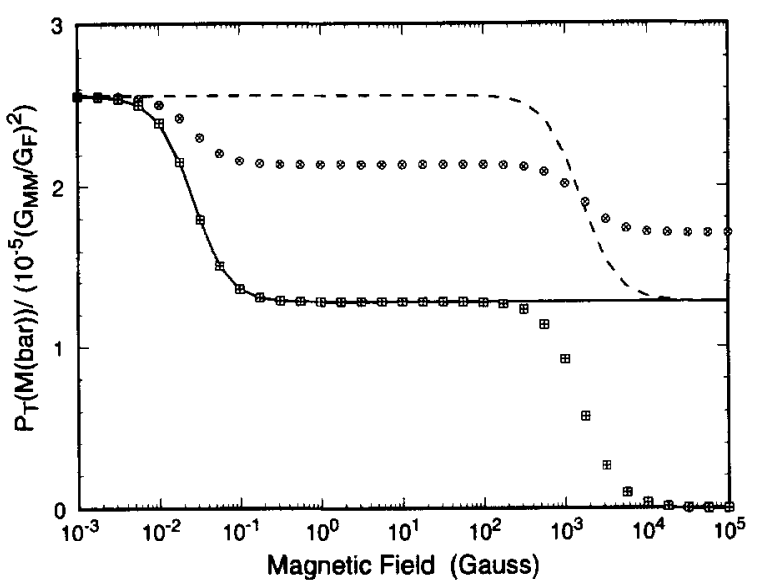

Fig. 1. Magnetic field dependence of total muonium conversion probability $P_{T}(\bar{M})$ assuming $\left|c_{1,1}\right|^{2}=\left|c_{1,0}\right|^{2}=\left|c_{1,-1}\right|^{2}=\left|c_{0,0}\right|^{2}$ $=1 / 4$, and normalized to conversion strength of $(V-A)(V-A)$ interaction at zero magnetic field. Solid and dashed lines stand for $S S$ and $P P$ operators, respectively, while $\circ, \square,+$ and $\times$ stand for $(S-P)(S+P),(S-P)(S-P),(V-A)(V+A)$ and $(V-A)(V-A)$ cases, respectively.

and all other matrix elements vanish. One therefore finds the result [13]

$$
\begin{aligned}
& P^{(1, \pm 1)}(\bar{M})=\frac{\bar{\delta}^{2}}{2\left(\bar{\delta}^{2}+a^{2} Y^{2}+\lambda^{2}\right)}, \\
& P^{(1, \quad 0)}(\bar{M})=P^{(0,0)}(\bar{M}) \\
& =\frac{\bar{\delta}^{2}}{2\left(\bar{\delta}^{2}+\lambda^{2}\left(1+X^{2}\right)\right)} .
\end{aligned}
$$

The magnetic field dependence for $P_{T}(\bar{M})$, as well as the separate probabilities $P^{(1, \pm 1)}(\bar{M}), P^{(1,0)}(\bar{M})$ and $P^{(0,0)}(\bar{M})$ of Eq. (17), are plotted as “+” symbols in Figs. 1-4, respectively. The behavior is readily understood. Because of the $a Y$ energy splitting, the suppression of $(1, \pm 1)$ modes sets in with $B$ field of just a few cG, and they become quenched for $0.1 \mathrm{G}$ or higher. The $m_{F}=0$ "unpolarized" modes are oblivious to the magnetic field until $X$ becomes appreciable, i.e. for $B \sim a / 2 \mu_{B} \sim 1 \mathrm{kG}$, and get quenched by fields of 1 Tesla or higher. The scale difference for Fig. 4 would be discussed shortly. The suppression in $P(\bar{M})$ has been taken into account in the experimental limit of Eq. (2) for the interaction of Eq. (1).

We have checked and confirmed the result for the $(V-A)(V+A)$ case [11], 


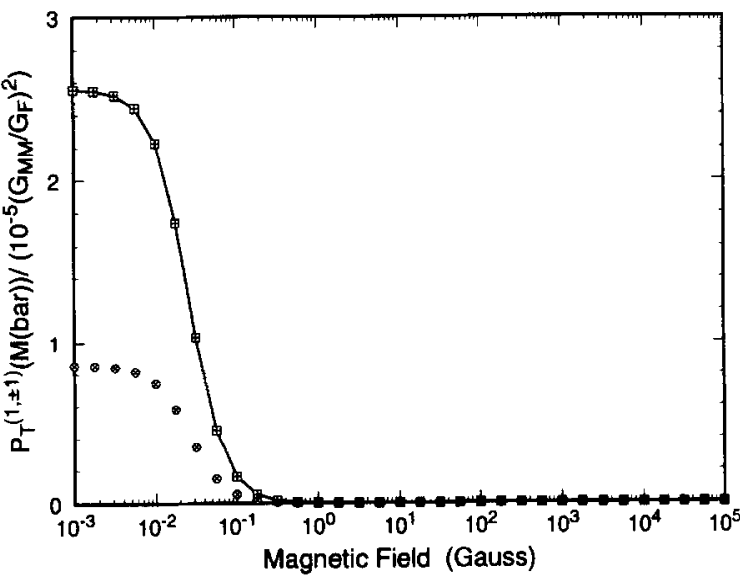

Fig. 2. $P^{(1, \pm 1)}(\bar{M})$ vs. magnetic field with same assumption as Fig. 1.

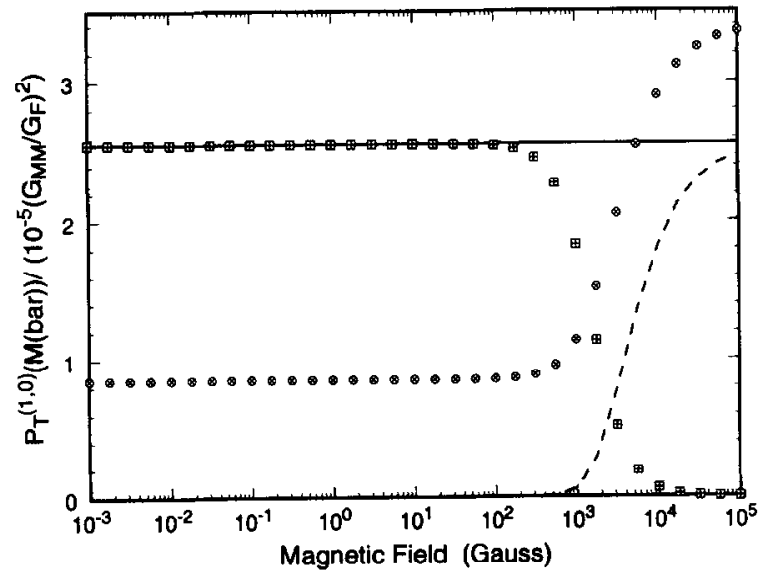

Fig. 3. $P^{(1,0)}(\bar{M})$ vs. magnetic field with same assumption as Fig. 1.

$$
\begin{gathered}
P^{(1, \pm 1)}(\bar{M})=\frac{\bar{\delta}^{2}}{6\left(\bar{\delta}^{2}+a^{2} Y^{2}+\lambda^{2}\right)} \\
P^{(1,0)}(\bar{M}), P^{(0,0)}(\bar{M}) \\
=\frac{\left(2 \mp \frac{1}{\sqrt{1+X^{2}}}\right)^{2} \bar{\delta}^{2}}{6\left[\left(2 \mp \frac{1}{\sqrt{1+X^{2}}}\right)^{2} \bar{\delta}^{2}+\lambda^{2}\right]}
\end{gathered}
$$

The results are also plotted in Figs. 1-4 as " $x$ " symbols. Conversion in $(0,0)$ mode is the most prominent [11], but gets suppressed by up to a factor of $4 / 9$ when the magnetic field goes beyond $\sim 1 \mathrm{kG}$. The $(1, \pm 1)$ modes are quenched by magnetic fields of

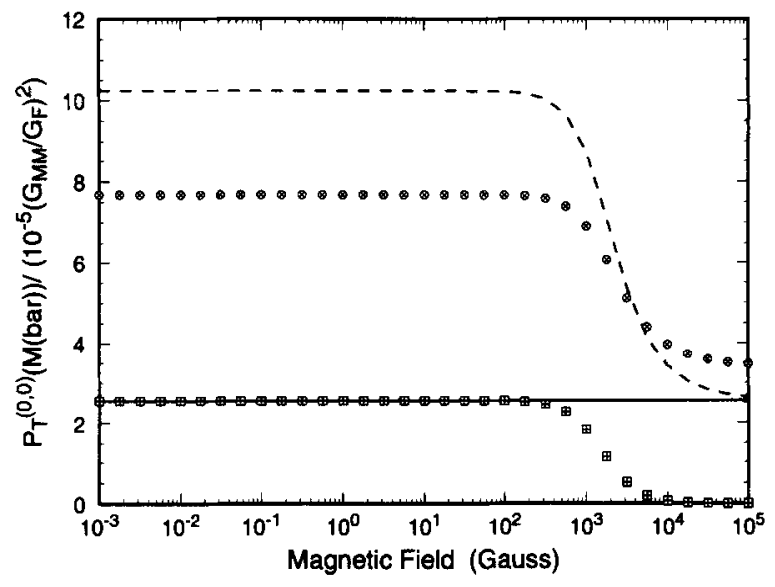

Fig. 4. $P^{(0,0)}(\vec{M})$ vs magnetic field with same assumption as Fig. 1.

$0.1 \mathrm{G}$ or higher, just like in the $(V-A)(V-A)$ case, but $P^{(1,0)}(\bar{M})$ actually grows with $B$ field around 1 $\mathrm{kG}$, and partially compensates for the drop in $P^{(0,0)}$.

Let us specify neutral (pseudo)scalar induced interactions [8]. Assuming $C P$ conservation, one has the effective Hamiltonian

$$
\begin{aligned}
\mathcal{H}_{M \bar{M}} & =\frac{f_{H}^{2}}{2 m_{H}^{2}} \bar{\mu} e \bar{\mu} e-\frac{f_{A}^{2}}{2 m_{A}^{2}} \bar{\mu} \gamma_{5} e \bar{\mu} \gamma_{5} e \\
= & \frac{1}{4}\left(\frac{f_{H}^{2}}{m_{H}^{2}}+\frac{f_{A}^{2}}{m_{A}^{2}}\right)\left(S^{2}-P^{2}\right) \\
+ & \frac{1}{4}\left(\frac{f_{H}^{2}}{m_{H}^{2}}-\frac{f_{A}^{2}}{m_{A}^{2}}\right)\left(S^{2}+P^{2}\right)
\end{aligned}
$$

where $S=\bar{\mu} e$ and $P=\bar{\mu} \gamma_{5} e$. Note that $S^{2}-P^{2}$ and $S^{2}+P^{2}$ contain $(S \mp P)(S \pm P)$ and $(S \mp P)(S \mp P)$ interactions, respectively. In the " $U(1)$ limit" of $f_{H}=$ $f_{A}$ and $m_{H}=m_{A}$ the subleading $(S \mp P)(S \mp P)$ terms are completely absent. The matrix elements of Eq. (19) have been discussed in Ref. [8], allowing us to give the results for the (pseudo) scalar induced interaction case. Details would be given elsewhere [14]. For purely scalar interactions $\left(f_{A}=0\right)$, we find

$$
\begin{aligned}
& P^{(1, \pm 1)}(\bar{M})=\frac{\bar{\delta}^{2}}{2\left(\bar{\delta}^{2}+a^{2} Y^{2}+\lambda^{2}\right)}, \\
& P^{(1,0)}(\bar{M})=P^{(0,0)}(\bar{M})=\frac{\bar{\delta}^{2}}{2\left(\bar{\delta}^{2}+\lambda^{2}\right)} .
\end{aligned}
$$

Thus, aside from the familiar quenching of the $(1, \pm 1)$ states, the $(1,0)$ and $(0,0)$ states are completely in- 
sensitive to magnetic fields [10]. For purely pseudoscalar interactions $\left(f_{H}=0\right)$, we find

$$
\begin{aligned}
& P^{(1, \pm 1)}(\bar{M})=0, \\
& P^{(1,0)}(\bar{M}), P^{(0,0)}(\bar{M}) \\
& =\frac{\left(\mp 1+\frac{1}{\sqrt{1+X^{2}}}\right)^{2} \bar{\delta}^{2}}{2\left[\left(\mp 1+\frac{1}{\sqrt{1+X^{2}}}\right)^{2} \bar{\delta}^{2}+\lambda^{2}\right]} .
\end{aligned}
$$

In this case, muonium conversion occurs solely in the $(0,0)$ mode for zero magnetic field [8]. Conversion in the $(1,0)$ mode starts to grow from zero for field strengths beyond $\sim 1 \mathrm{kG}$, and partially compensates for the drop, by a factor of 4 , in transition probability in the $(0,0)$ mode. We plot the results again in Figs. $1-4$, with solid and dashed lines representing scalar and pseudoscalar case, respectively.

The results for $(S-P)(S-P)$ and $(S-P)(S+P)$ operators can be similarly obtained. With the same normalization conditions as described above, the results are also given in Figs. 1-4 for sake of comparison, with open circles representing the $(S-P)(S+P)$ case and open boxes representing the $(S-P)(S-P)$ case. Note that according to Eq. (19), the $(S-P)(S-P)$ operators should be subdominant compared to $(S-$ $P)(S+P)$ operators. Pure $(S \mp P)(S \pm P)$ operators correspond to complex neutral scalars [8], where the sneutrino $\tilde{\nu}_{\tau}$ in SUSY models with $R$-parity breaking [15] as a special case. It is evident from Figs. 1-4 that the combinations $(S-P)(S-P)$ and $(S-P)(S+P)$ behave in the same way as $(V \quad A)(V \quad A)$ and $(V-A)(V+A)$, respectively. In the latter case, the two operators are related to each other by a Fierz transform. For the former case, although the operators can not be related to each other by a Fierz transform, the matrix elements are always in same proportion, which comes as a consequence of the nonrelativistic limit.

The assumption of cqually populated Brcit-Rabi levels is not a valid one, since this is determined by the muonium formation process and the magnetic field strength [4]. However, as a consequence of this assumption, the results in Eqs. (17), (18) and (20), (21) and hence Figs. 2-4 all have an artificial factor of 4. To illustrate the effect of differently populated BreitRabi levels, normalizing again to the $(V-A)(V-A)$ case at zero $B$ field, we take muonium states to be populated as [4] $32 \%, 35 \%, 18 \%$ and $15 \%$, respectively,

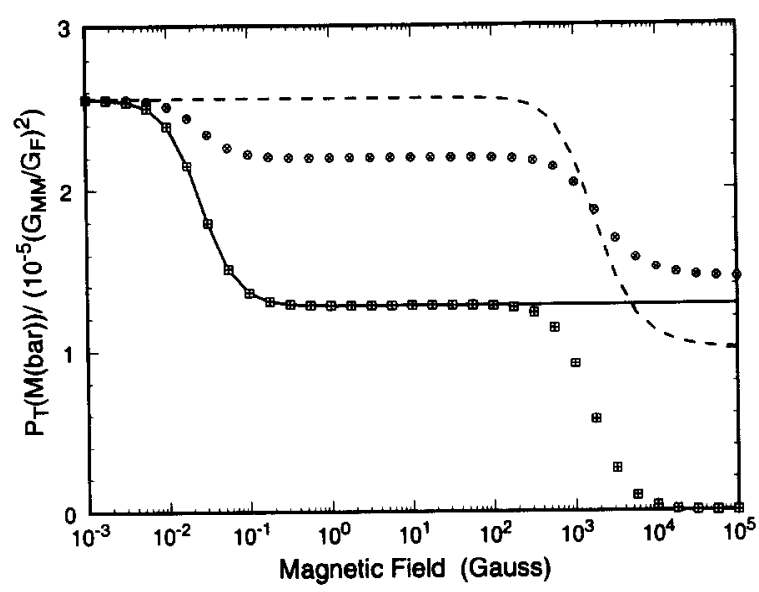

Fig. 5. The same as Fig. 1 except $\left|c_{1,1}\right|^{2}=0.35,\left|c_{1,0}\right|^{2}=0.18$, $\left|c_{1 .-1}\right|^{2}=0.15$ and $\left|c_{00}\right|^{2}=0.32$.

for $\left(F, m_{F}\right)=(0,0),(1,+1),(1,0),(1,-1)$, and plot the results for $P_{T}(\bar{M})$ (i.e. analogous to Fig. $1)$ in Fig. 5. The $(V-A)(V-A)$ and $(S-P)(S-P)$ results are unchanged, since the conversion matrix elements are the same for all modes. The purely scalar (SS) case is also unchanged, since $\left|c_{10}\right|^{2}+\left|c_{00}\right|^{2}=$ $\left|c_{1+1}\right|^{2}+\left|c_{1-1}\right|^{2}=50 \%$ is the same as the equally populated case. The $(V-A)(V+A),(S-P)(S+P)$ and $P P$ cases are somewhat modified from the equally populated case, but the difference for $1 \mathrm{kG}$ field is rather slight.

Let us summarize our findings. Purely neutral scalar (SS) induced $M-\bar{M}$ transitions in polarized (i.e. $\left.\left(F, m_{F}\right)=(1, \pm 1)\right)$ modes are quenched by magnetic fields of 0.1 Gauss or higher, but conversion in unpolarized states (i.e. $(1,0)$ and $(0,0))$ are independent of magnetic field strength. Purely pseudoscalar $(P P)$ interactions do not induce conversion in $(1, \pm 1)$ states, but exhibit compensating effects in the $(1,0)$ and $(0,0)$ channels, similar to the $(V-A)(V+A)$ case. Interactions of $(S \mp P)(S \pm P)$ and $(V \mp A)(V \pm A)$ form have the same magnetic field dependence since they are related by Fierz transform, while $(S \mp P)(S \mp P)$ and $(V \mp A)(V \mp A)$ interactions have the same field dependence because of proportional conversion matrix elements. Experimental search for $M-\bar{M}$ conversion as a probe of lepton number violation continues to be vigorously pursucd. Our results are useful for the interpretation of experimental limits for various effective interactions. Because of strongest quenching for $10^{3}$ Gauss 
field or higher, bounds on effective couplings are in fact the least stringent for the usual $(V-A)(V-A)$ interaction.

We thank K. Jungmann for numerous communications and a copy of Ref. [10]. The work of WSH is supported by grant NSC 84-2112-M-002-011, and GGW by NSC 84-2811-M-002-035 of the Republic of China.

\section{References}

11] G. Feinberg and S. Weinberg, Phys. Rev. Lett. 6 (1961) 381; Phys. Rev. 123 (1961) 1439.

12] B.E. Matthias et al., Phys. Rev. Lett. 66 (1991) 2716; V.A. Gordeev et al., JETP Lett. 59 (1994) 589.

[3] K. Jungmann et al., presented at 23rd INS "International Symposium on Nuclear and Particle Physics with Meson Beams in the 1-GeV/c Region", Tokyo, Japan, March 15-18, 1995.
[4] K. Jungmann et al., PSI Experiment No. R-89-06.1.

[5] P. Herczeg and R.N. Mohapatra, Phys. Rev. Lett. 69 (1992) 2475 , and references therein.

[6] H. Fujii, S. Nakamura and K. Sasaki, Phys. Lett. B 299 (1993) 342;

H. Fujii et al., Phys. Rev. D 49 (1994) 559.

[7] S. Adler, Phys. Lett. B 225 (1989) 143;

P.H. Frampton and B.-H. Lee, Phys. Rev. Lett. 64 (1990) 619.

[8] W.S. Hou and G.G. Wong, preprint NTUTH-95-03, April 1995.

[9] E. Derman, Phys. Rev. D 19 (1979) 317.

[10] Neutral scalar exchange is also mentioned by B.E. Matthias, $\mathrm{Ph} . \mathrm{D}$. thesis (LA-12202-T, 1991), with some discussion of difference in magnetic field dependence.

[11] K. Horikawa and K. Sasaki, preprint YNU-HEPTh-107, March 1995.

[12] See, for example, V.W. Hughes, Ann. Rev. Nucl. Sci. 16 (1966) 445 , or Ref. [10].

[13] W. Schäfer, Ph.D. Thesis (1988), Universität Heidelberg.

[14] W.S. Hou, T.C. Lo and G.G. Wong, in preparation.

[15] A. Halprin and A. Masiero, Phys. Rev. D 48 (1993) R2987. 